Scientific Journal of Hamadan Nursing \& Midwifery Faculty - ISSN 2008-2819

\title{
The Relationship Between Stress, Anxiety and Depression With Menopausal Women Experiences
}

\section{Sedigheh Shariat Moghani ${ }^{1}$, Masoumeh Simbar ${ }^{2 *}$, Farzaneh Rashidi Fakari ${ }^{3}$, Vida Ghasemi ${ }^{3}$, Mahrokh Dolatian ${ }^{4}$, Nahid Golmakani ${ }^{5}$, Malihe Nasiri ${ }^{6}$}

1. Faculty Member, Nursing and Midwifery Care Research Center, Mashhad University of Medical Sciences, Mashhad, Iran

2. Professor, Midwifery and Reproductive Health Research Center, School of Nursing and Midwifery, Shahid Beheshti University of Medical Sciences, Tehran, Iran

3. PhD Student of Reproductive Health, Students Research Committee, Department of Midwifery and Reproductive Health, School of Nursing and Midwifery, Shahid Beheshti University of Medical Sciences, Tehran, Iran

4. Assistant Professor, Obstetrics and Gynecology Research Center, School of Nursing and Midwifery, Shahid Beheshti University of Medical Sciences, Tehran, Iran

5. Assistant Professor, Nursing and Midwifery Care Research Center, Mashhad University of Medical Sciences, Mashhad, Iran

6. Department of Biostatistics, School of Allied Medical Sciences, Shahid Beheshti University of Medical Sciences, Tehran, Iran

Article Info $\quad$ Abstract

Received: $\quad 2017 / 07 / 31$

Accepted: $\quad 2017 / 11 / 7$

Published Online: 2017/11/18

DOI:

10.30699/sjhnmf.26.a5.333

Original Article

Use your device to scan and read the article online

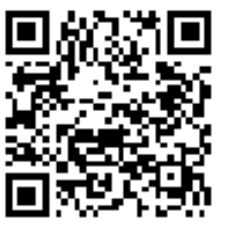

Introduction: Women spend more than a third of their lives in menopause and estrogen deficiency. These neuroendocrine changes affect the woman's sense of health and mental health through the loss of fertility and menstrual function. Therefore, the present study was conducted to investigate the association between anxiety, stress and depression with women's experiences in menopause.

Method: This was a descriptive-correlation study conducted on 250 postmenopausal women who met the inclusion criteria in 2016 in Mashhad. A convenience quota sampling method was used. The data gathering tool was a demographic questionnaire, DASS21, measuring the experiences of menopause women. Data analysis was performed using SPSS 16 software by Pearson correlation coefficient and regression model at a significant level of 0.05 .

Results: The mean age of menopause was $48 \pm 4.56$ years. The mean and standard deviation of anxiety score, stress and depression in postmenopausal women were $5.43 \pm 4.8,4.37 \pm 7.7$ and $4.33 \pm 5.35$, respectively. The mean total score of menopausal experiences was $33.5 \%$. Pearson correlation showed a direct and significant association between stress, anxiety and depression score with the experiences of women in menopause $(P=0.001, \mathrm{r}=0.68)$.

Conclusion: with increasing the scores of depression, anxiety and stress, the score of women's experiences in menopause increased. Therefore, these changes require counseling and supportive program to improve adaptation to menopausal psychosocial changes.

Keywords: Menopause, Experiences, Anxiety, Stress, Depression

Masoumeh Simbar, Professor, Midwifery and Reproductive Health Research Center, School of Nursing and Midwifery, Shahid Beheshti University of Medical Sciences, Tehran, Iran. Email: simbarm@gmail.com

Corresponding Information

Copyright (C) 2018, Sci J Hamadan Nurs Midwifery Fac. This is an open-access article distributed under the terms of the Creative Commons Attribution-noncommercial 4.0 International License which permits copy and redistribute the material just in noncommercial usages, provided the original work is properly cited.

How to Cite This Article:

Shariat Moghani S, Simbar M, Rashidi Fakari F, Ghasemi V, Dolatian M, Golmakani N, et al . The Relationship Between Stress, Anxiety and Depression With Menopausal Women Experiences. Sci J Hamadan Nurs Midwifery Fac. 2018; 26 (5):333-340 
مجله علمى دانشكدهُ يرستارى و مامايى همدان - شايا الكترونيك: 19

مقاله يزوهشى

ارتباط اضطراب، استرس و افسردَى با تجارب زنان در يائسخىى

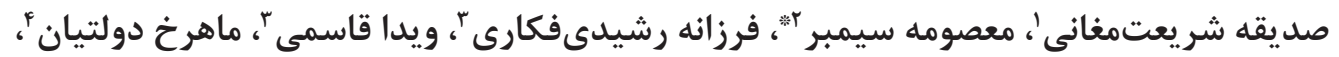

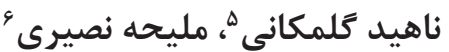

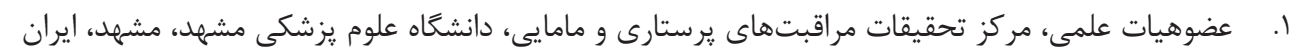

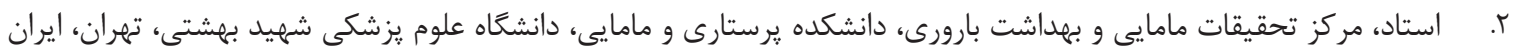

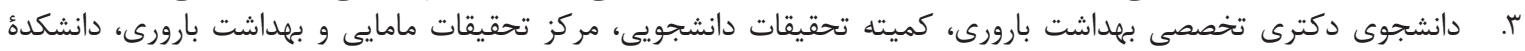

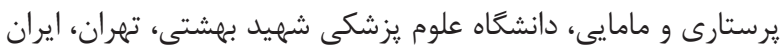

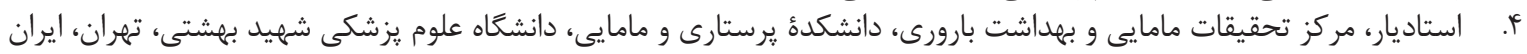

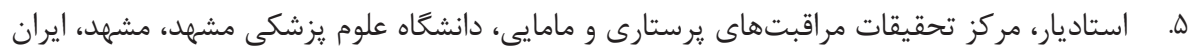

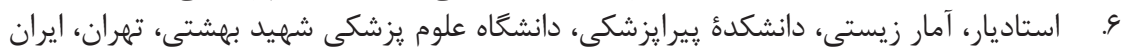

\begin{tabular}{|c|c|}
\hline جكيده & اطلاعات مقاله \\
\hline 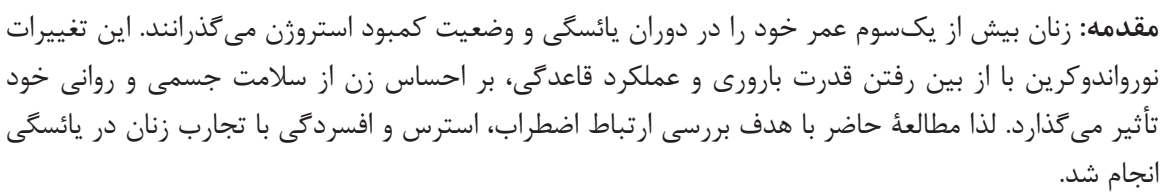 & تاريخ وصول: $\quad$ تاريخ \\
\hline 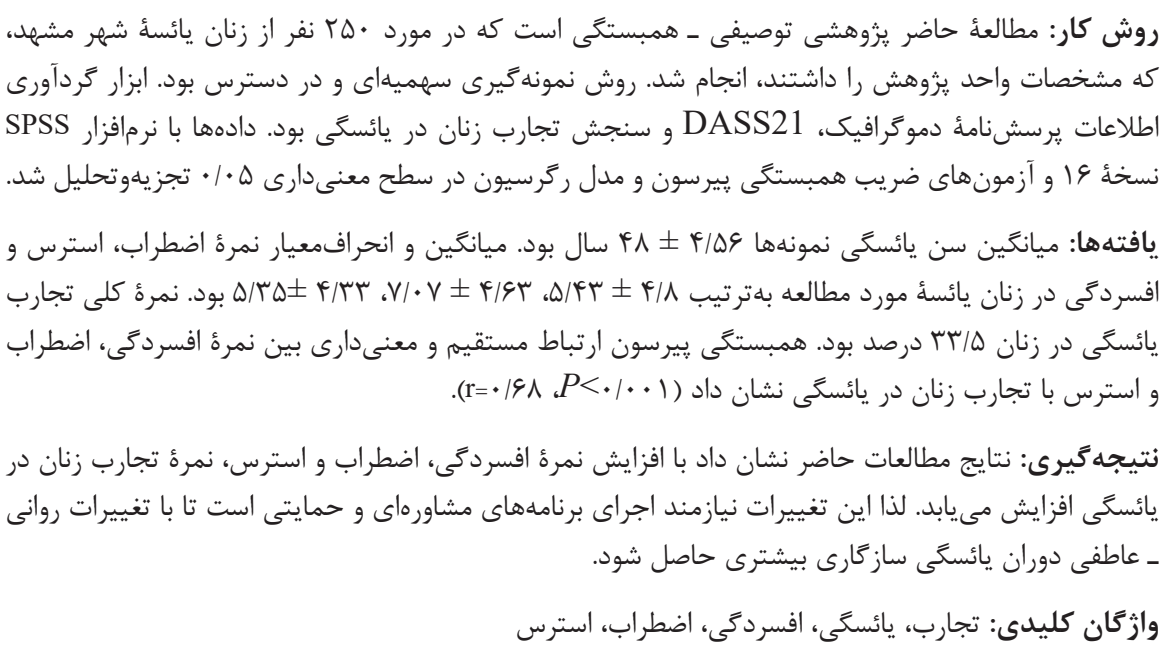 & 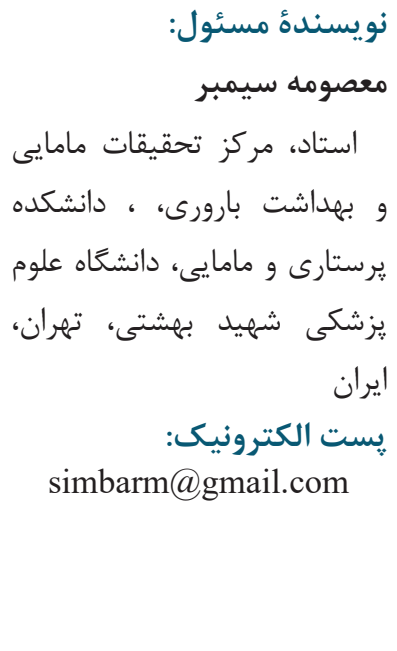 \\
\hline
\end{tabular}

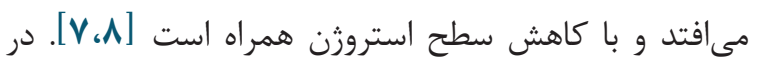
مقلممه

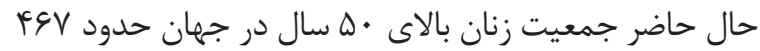

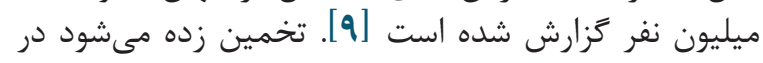

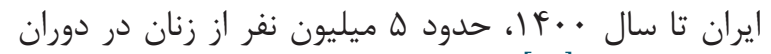

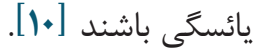

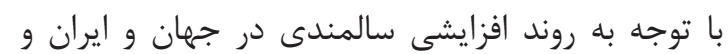

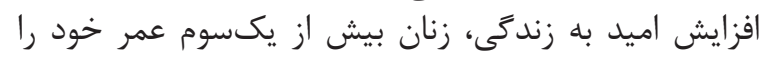

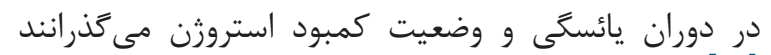

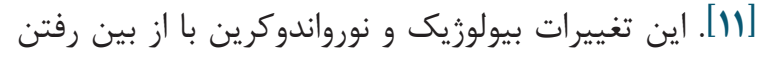

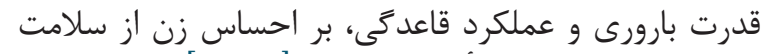

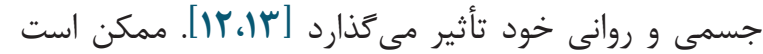

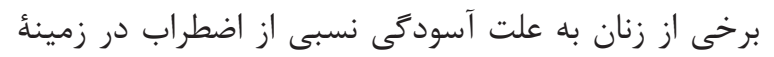

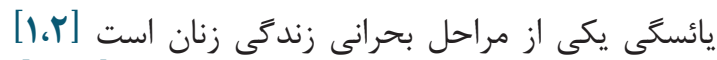

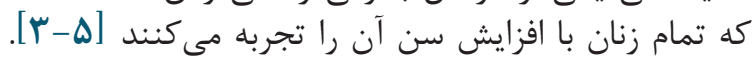

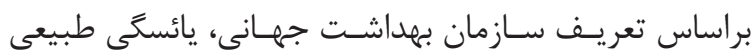

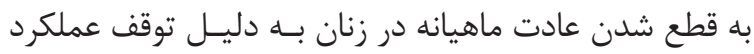

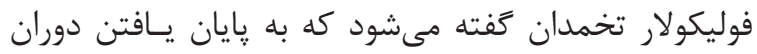

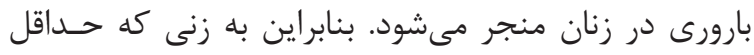

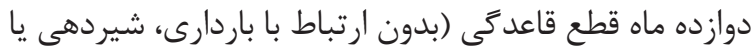

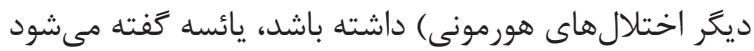

يائسگى بهطور معمول در زنان بالاى •ه سال اتفاق 
يُ إز از تقسيم شهر مشهد به مناطق ينج كانه (شمال، شرق،

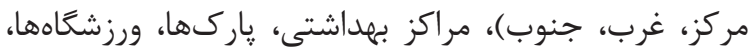

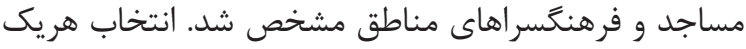

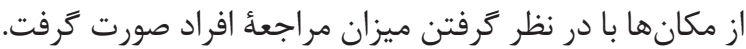

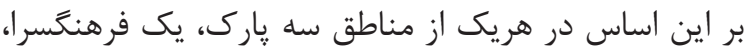

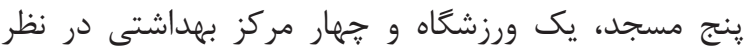

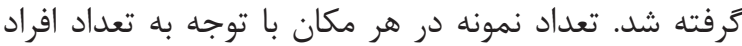

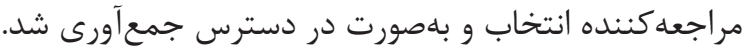

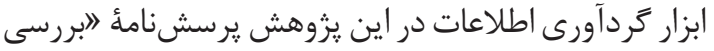

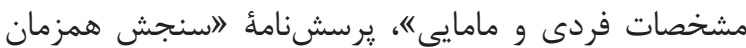

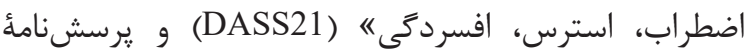

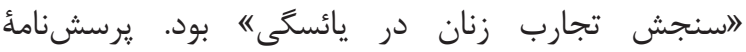

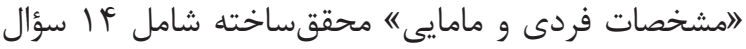

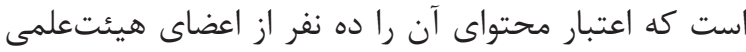

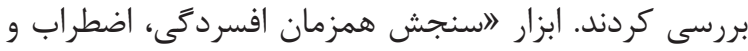

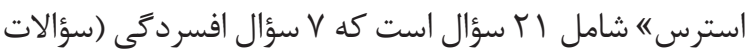

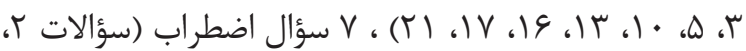

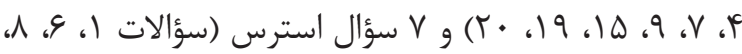

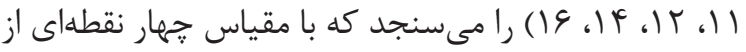

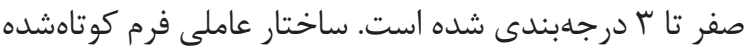

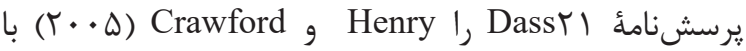

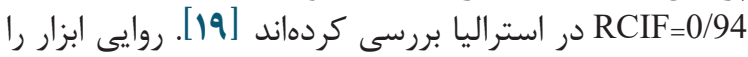
در ايران Sahebi و همكاران (

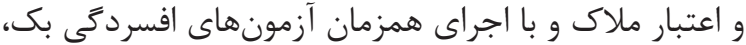

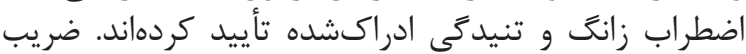

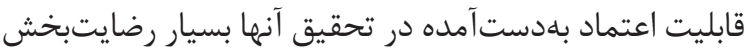

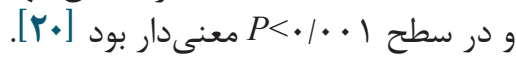

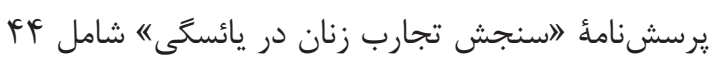

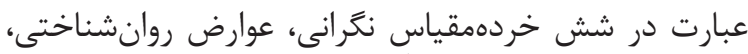

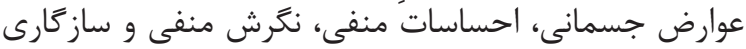

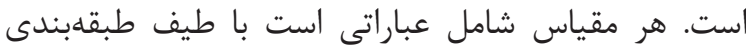

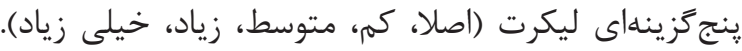

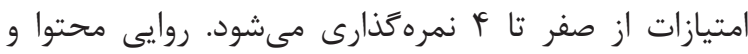

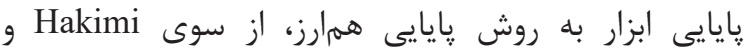

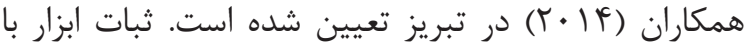

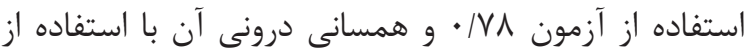

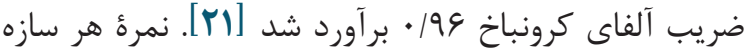

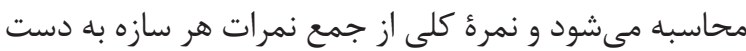

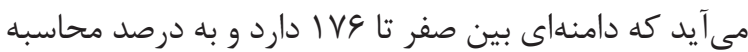

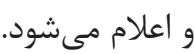

يزوهشكَ بعد از معرفى خود به زنان انتخابشده، دربارئ

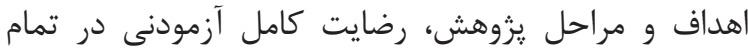

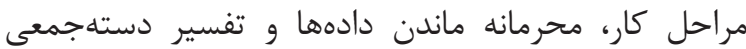

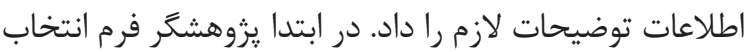

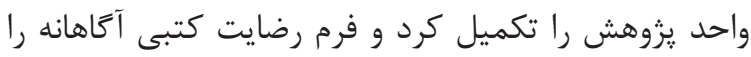

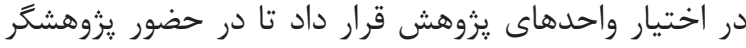

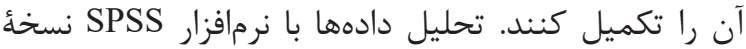

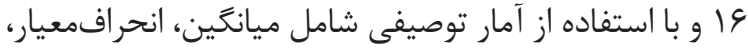

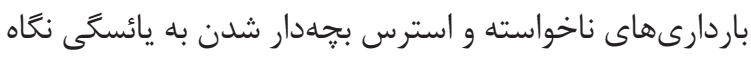

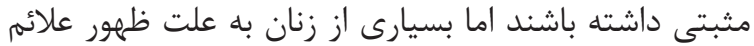

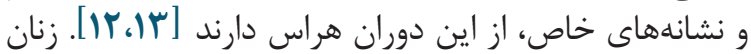

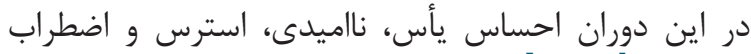

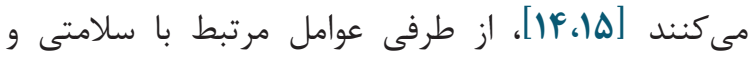

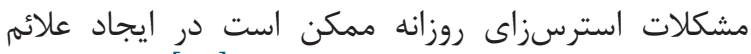
افسردگى در اين دوران نقش داشته باشن داشد [19].

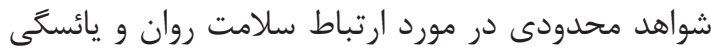

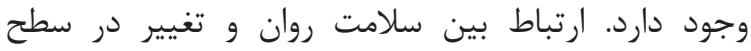

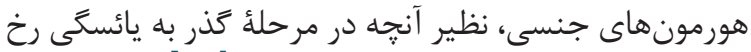
مى دهد، هنوز موضوعى بحثبرانگيز است [IV]

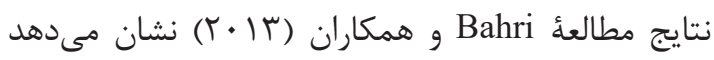

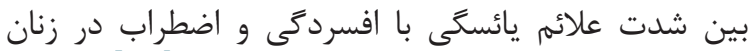

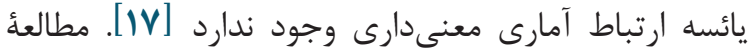

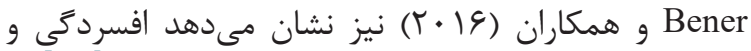

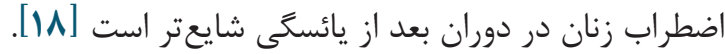
لذا با توجه به محدود و متناقض بودن مطالعات انجامشده

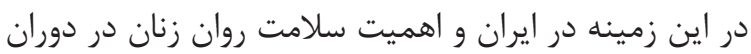

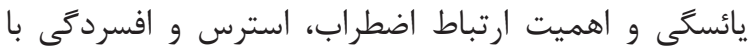

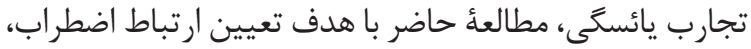

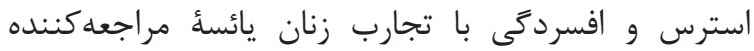

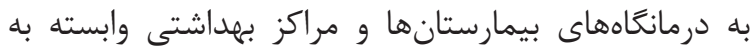

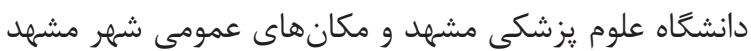

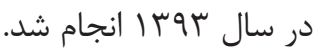

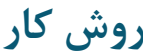

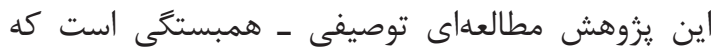

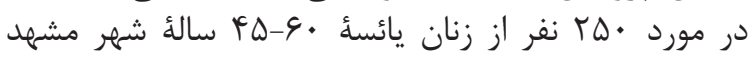

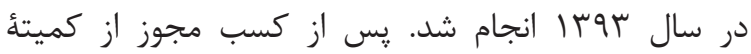

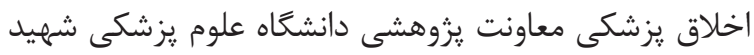

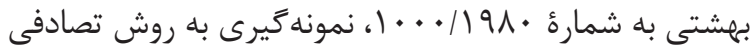

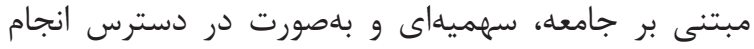

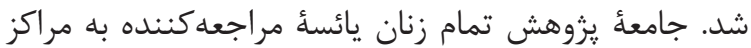

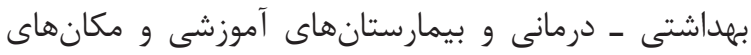

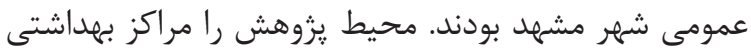

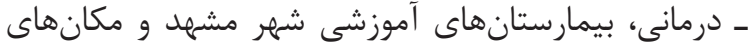

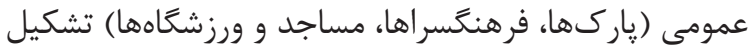

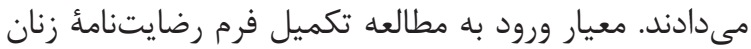

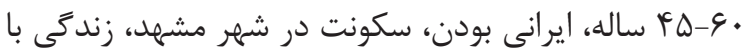

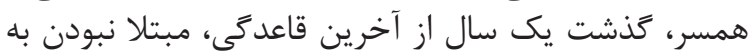

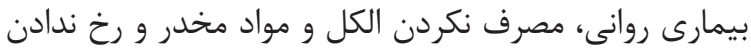

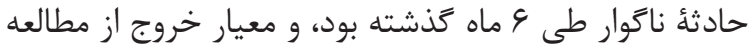

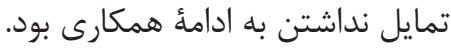
حجم نمونه با احتساب توان •1 درصد و فاصلئ اطمينان

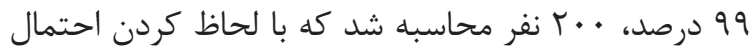

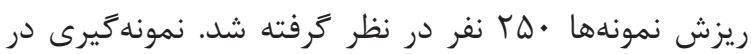

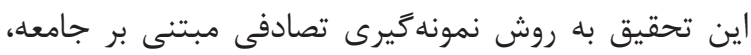

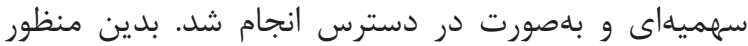


زنان بودند. حدود 1/

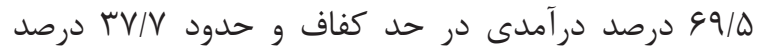

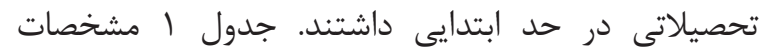
جمعيتشناختى شركت كنند مى جدهد.
توزيع فراوانى مطلق و نسبى و آمـار تحليلى شامل ضريب

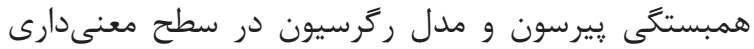

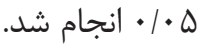

بافتهها.

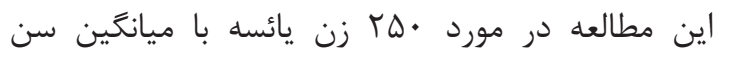

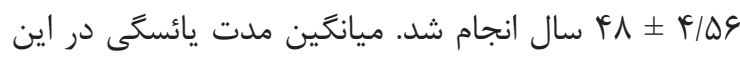

جدول ا. مشخصات جمعيتشناختى زنان يائسهُ شركت كننده در يروهش

\begin{tabular}{|c|c|c|}
\hline (درصد) تعداد & & \\
\hline$f(1 / \Lambda)$ & كارگر & \multirow{5}{*}{ شغل } \\
\hline$r q(1 r / r)$ & كارمند & \\
\hline $109(V Y / T)$ & خانهدار & \\
\hline$r \varphi(1) / \Lambda)$ & بازنشسته & \\
\hline$r(\cdot / 9)$ & شاغل خانكى & \\
\hline $\mid \wedge \Delta(\wedge F / \mid)$ & 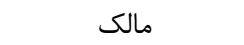 & \multirow{3}{*}{ وضعيت مسكن } \\
\hline $\operatorname{rF}(\mid \Delta / \Delta)$ & استيجارى & \\
\hline $1(\cdot / \theta)$ & زندكى با خويشاوندان & \\
\hline $\operatorname{FF}(Y \cdot)$ & كمتر از حد كفاف & \multirow{3}{*}{ در آمد ماهيانه } \\
\hline $1 \Delta r(99 / \Delta)$ & در حد كفاف & \\
\hline$r r(1 \cdot \mid \Delta)$ & بيشتر از حد كفاف & \\
\hline $\mid r(\Delta / \Delta)$ & بىسواد & \multirow{5}{*}{ تحصيلات زنان } \\
\hline$\Lambda r(r V / V)$ & ابتدايى & \\
\hline$r r(\mid F / D)$ & سيكل & \\
\hline$F q(Y Y / T)$ & متوسطه & \\
\hline$F F(T \cdot)$ & عالى & \\
\hline
\end{tabular}

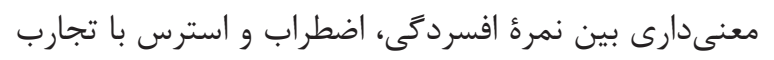

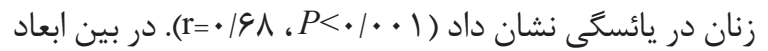

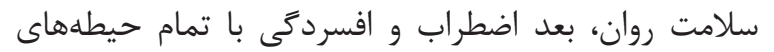

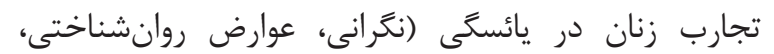

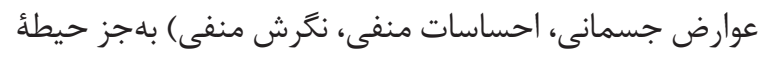

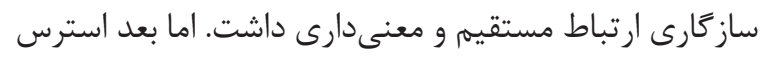

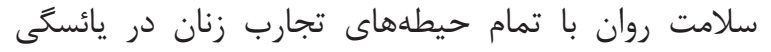

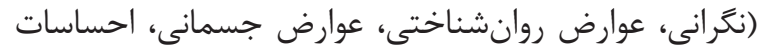

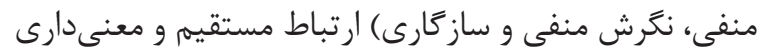
داشت.

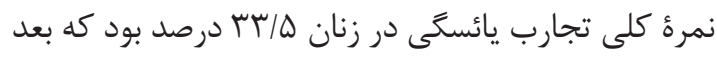

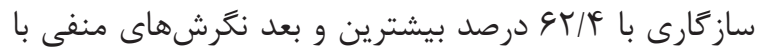


جدول ץ ميانگين و انحرافمعيار نمرات سلامت روان

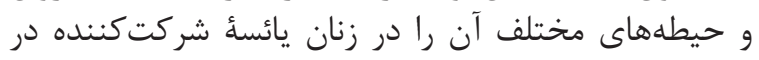

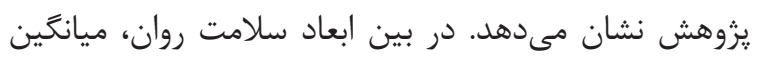

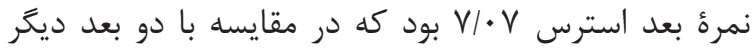

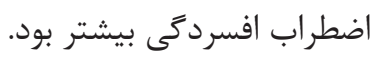
جدول ^ا ميزان همبستخى ميان سلامت روان و ابعاد آن آن

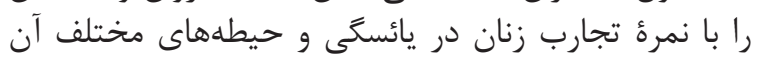

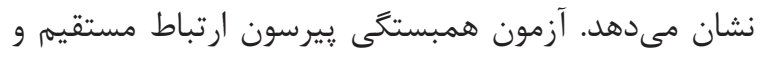


معصومه سيمبر و همكاران

جدول r. ميانغين و انحرافمعيار نمرات تجارب زنان در يائسكى و ابعاد آن بر مبناى ..1

\begin{tabular}{|c|c|c|}
\hline انحرافمعيار & ميانتين & تجارب زنان در يائسكَى \\
\hline rq/V & $G T / F$ & سازگَارى \\
\hline$r \varepsilon / F$ & MF/G & احساسات منفى \\
\hline $19 / 0$ & $M F / D$ & عوارض جسمانى \\
\hline$r \mid / r$ & 童 & عوارض روانشناختى \\
\hline$r y / 9$ & $r q / r v$ & ن نكرانى \\
\hline$r \varepsilon / Q$ & $T V / Q$ & نخرش هاى منفى \\
\hline $11 / 11$ & 要 & كل \\
\hline
\end{tabular}

جدول r. ميانگين و انحر افمعيار نمرات سلامت روان و حيطههاى مختلف آن در زنان يائسهُ

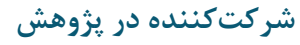

\begin{tabular}{|c|c|c|c|}
\hline محدودة نمره & انحرافمعيار & ميانكين & سلامت روان \\
\hline$\cdot-r_{1}$ & $F / \Lambda$ & $D / F T$ & اضطراب \\
\hline$\cdot-r_{1}$ & F/gT & $V / \cdot V$ & استرس \\
\hline$\cdot-r_{1}$ & س & $\Delta / \Gamma \Delta$ & افسردگى \\
\hline - - & $\mid K / g r$ & $1 N / T \Delta$ & كل \\
\hline
\end{tabular}

جدول F. ميزان همبستكى نمرات ابعاد سلامت روان با نمرات حيطههاى تجارب زنان در يائسكى

\begin{tabular}{|c|c|c|c|c|c|c|c|}
\hline كل & سازَارى & نَرش منفى & احساسات منفى & جسمانى عواض & روانشناختى عوارض & نقَرانى & سبقات \\
\hline $\begin{array}{c}* * * / \Delta T \Lambda \\
\cdot / \cdot \cdots\end{array}$ & $\begin{array}{l}\cdot \text {. AT } \\
\text {. KTE }\end{array}$ & $\begin{array}{c}* * * / \sigma \mid \Delta \\
\cdot / \ldots\end{array}$ & $\begin{array}{c}* *|\Delta \varphi| \\
\cdot \mid \cdots\end{array}$ & $\begin{array}{c}* * * / 4 \cdot V \\
\cdot / \cdot \cdots\end{array}$ & $\begin{array}{c}* * 1099 \\
\cdot / \ldots\end{array}$ & $\begin{array}{c}* * * T V \mid \\
\cdot / \ldots\end{array}$ & اضطراب \\
\hline $\begin{array}{c}* * / \Delta \wedge r \\
\cdot / \cdot \cdots\end{array}$ & $\begin{array}{l}* . / l F T \\
. / . r q\end{array}$ & $\begin{array}{c}* * * / r q \Lambda \\
\cdot / .\end{array}$ & $\begin{array}{c}* * *|\Delta \wedge| \\
\cdot / \ldots\end{array}$ & $\begin{array}{c}* * / F F V \\
\cdot / \cdot\end{array}$ & $\begin{array}{c}* * \cdot|9| \mathrm{V} \\
\cdot / \cdot \cdots\end{array}$ & $\begin{array}{c}* * / F T f \\
\cdot / \cdots\end{array}$ & استرس \\
\hline $\begin{array}{c}* * / \Delta \vee q \\
\cdot / \cdot \cdots\end{array}$ & $\begin{array}{l}. / 11 \mathrm{~V} \\
. / \cdot 14\end{array}$ & $\begin{array}{c}* * * / 4 r T \\
. /\end{array}$ & $\begin{array}{c}* * / 010 \\
. / \cdots\end{array}$ & $\begin{array}{c}* * / Q \mid f \\
\cdot / \cdots\end{array}$ & $\begin{array}{c}* * \cdot \mid \Delta \& \Lambda \\
\cdot / \cdots\end{array}$ & $\begin{array}{c}* * / 4 r . \\
\cdot / \cdot \ldots\end{array}$ & افسردگى \\
\hline $\begin{array}{c}* * 19 \Lambda . \\
\cdot / \cdots\end{array}$ & $\begin{array}{l}* \cdot / 1 \Delta \Delta \\
\cdot|\cdot r|\end{array}$ & $\begin{array}{c}* * / 4 \wedge V \\
\cdot / \cdots\end{array}$ & $\begin{array}{c}* * \cdot 9 \Delta f \\
\cdot / .\end{array}$ & $\begin{array}{c}* * \cdot \Delta \Delta \mid \\
\cdot / \cdots\end{array}$ & $\begin{array}{c}* * / V \cdot \Delta \\
\cdot / \cdots\end{array}$ & $\begin{array}{c}* * / 499 \\
\cdot / .\end{array}$ & كل \\
\hline
\end{tabular}




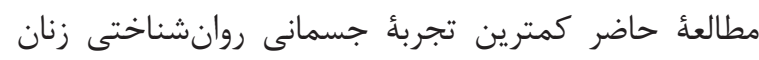

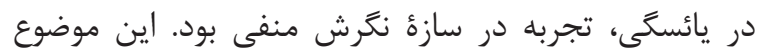

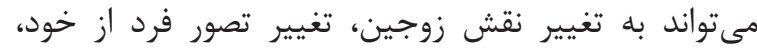

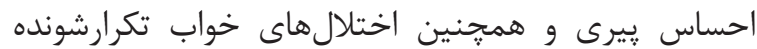

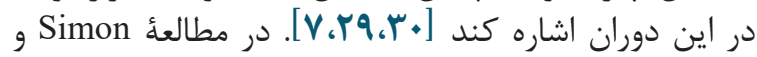
بائه مeape

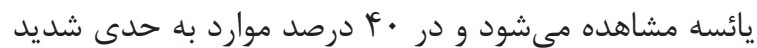

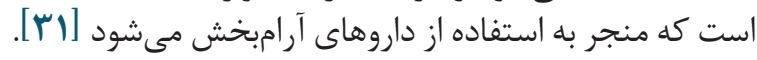

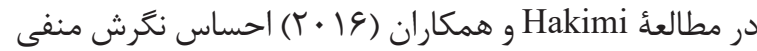

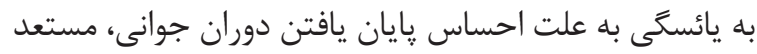

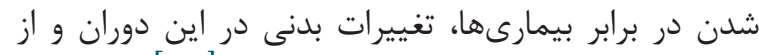

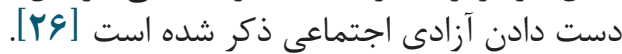

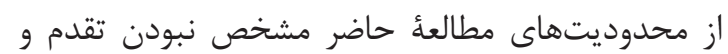

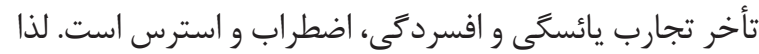

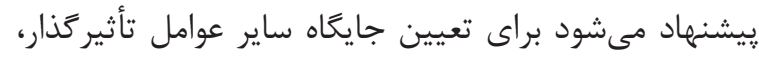

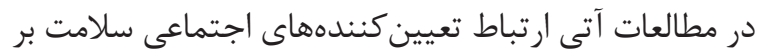
تجارب يائسكى در زنان بررسى شود.

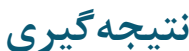

نتايج مطالعه حاضر نشان داد كه در بين حيطه هاى تجارب

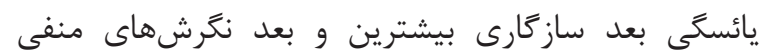

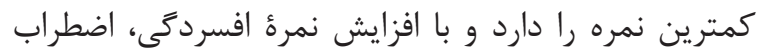

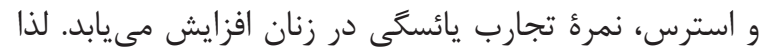

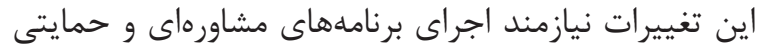
بابه منظور بهبود سازكارى با تغييرات روانى - عاطفى در زنان انيان

يائسه است.

\section{سياسگَزارى}

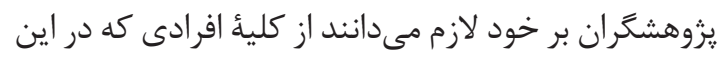

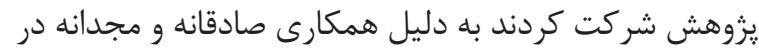

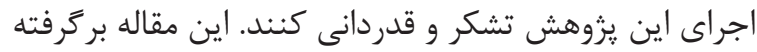

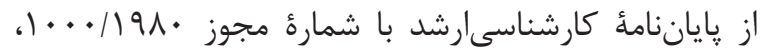

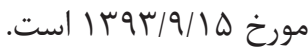

\section{تعارض منافع}

سهم تمام نويسندكان در اين مطالعه يكسان است و هيجَّونه تضاد منافعى وجود ندارد.

\section{بحث}

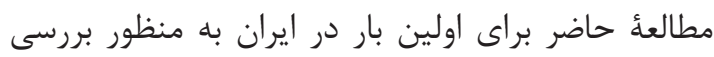

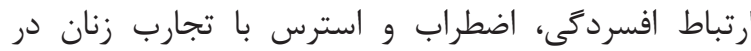

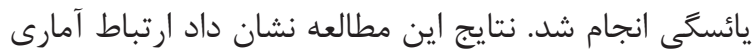

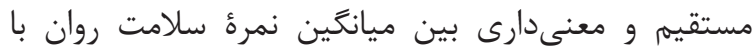

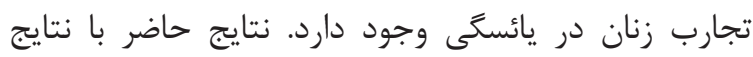

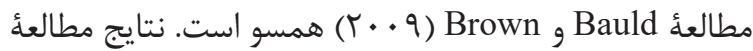

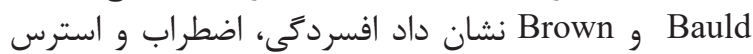

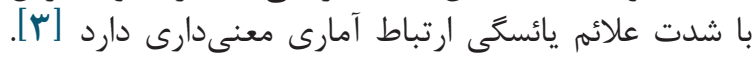

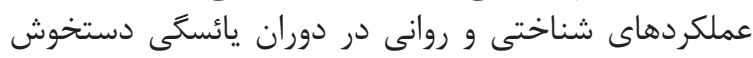

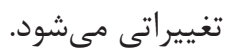

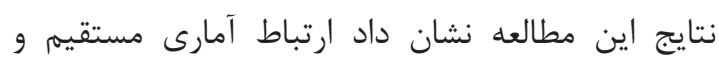

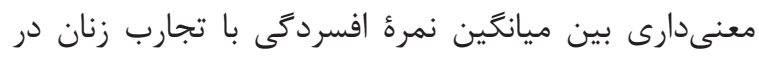

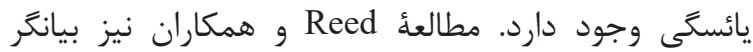

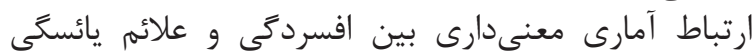

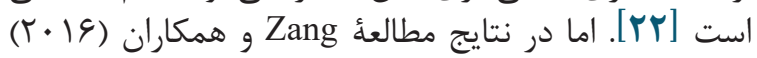

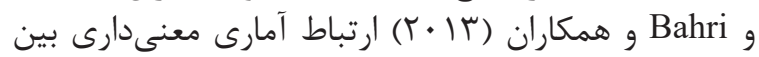

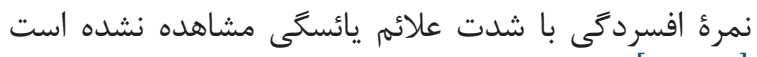

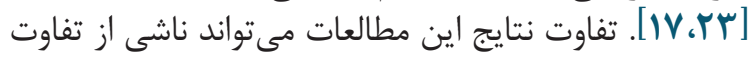

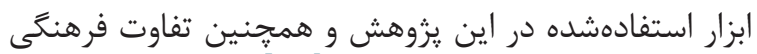

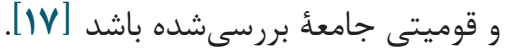

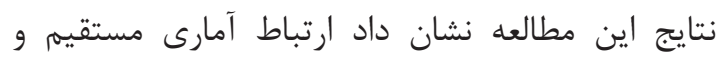

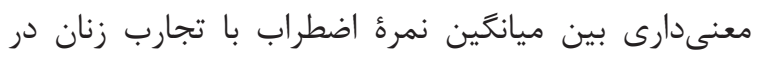

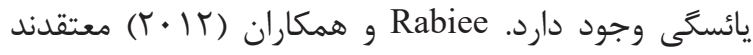

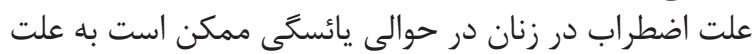

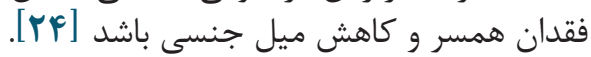

براساس يافتههاى اين يزوهش، ارتباط آمارى مستقيم

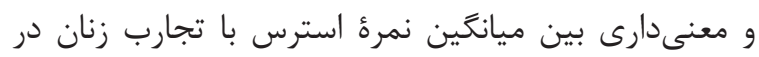

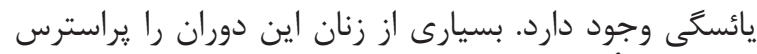

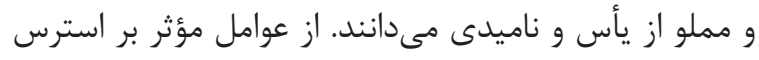

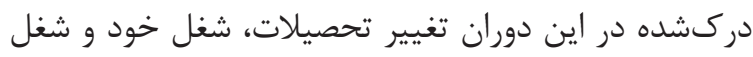

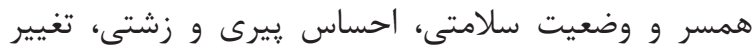

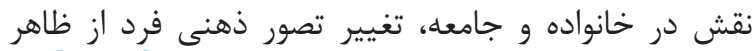

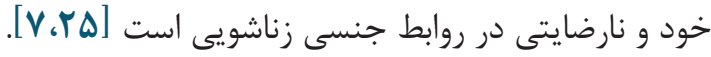

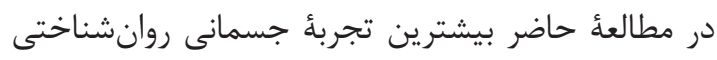

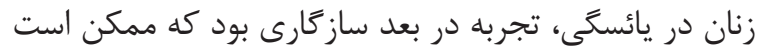

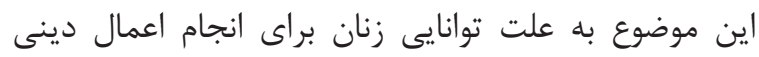

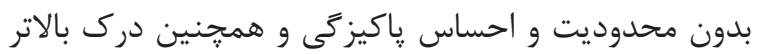

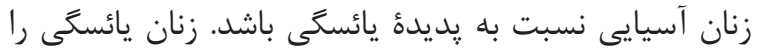

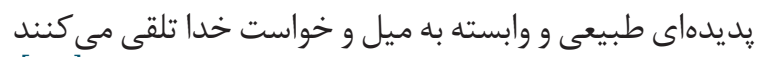

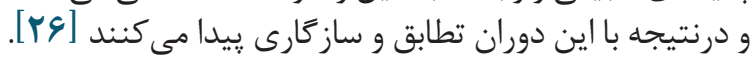

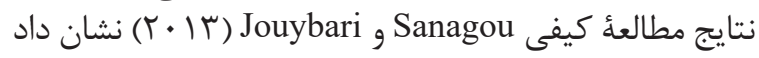

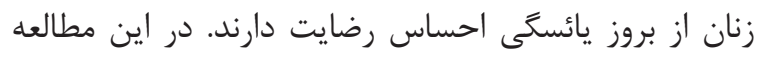

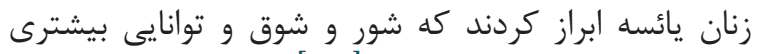

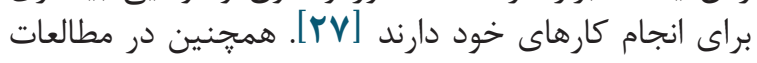

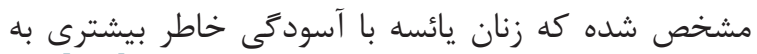

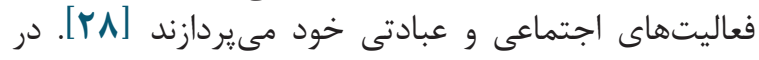




\section{References}

1. Abernethy K, Tiran D. The menopause and HRT: Elsevier Health Sciences; 2002.

2. Mattox JH. Core Textbook of Obstetrics and Gynecology: Mosby Incorporated; 1998.

3. Bauld R, Brown RF. Stress, psychological distress, psychosocial factors, menopause symptoms and physical health in women. Maturitas. 2009;62(2):160-5.https://doi.org/10.1016/j.maturitas.2008.12.004. PMID:19167176

4. Novak E, Berek JS. Be+rek \& Novak's gynecology: Lippincott Williams \& Wilkins; 2007.

5. Zöllner Y, Acquadro C, Schaefer M. Literature review of instruments to assess health-related quality of life during and after menopause. Quality of Life Research. 2005;14(2):309-27. https://doi.org/10.1007/s11136-004-0688-z PMID: 15892422

6. Sadat Hashemi S M, Ghorbani R, Kavhie B, Askari Majdabadi H, Shadmehr A. Non-parametric estimation of the pattern of the age at natural menopause in Garmsar. koomesh. 2008; 9 (2):139-146.

7. Afghari A, Ahmad Shirvani M. Psycho-emotional changes in menopause: A qualitative study. J Mazandaran Univ Med Sci. 2012;22(93):27-38.

8. Nazarpour S, Simbar M, Tehrani FR. Factors affecting sexual function in menopause: A review article. Taiwanese Journal of Obstetrics and Gynecology. 2016;55(4):480-7. https://doi. org/10.1016/j.tjog.2016.06.001 PMID:27590367

9. Speroff L, Fritz MA. Clinical gynecologic endocrinology and infertility: lippincott Williams \& wilkins; 2005.

10. Ministry of Health and Medical Education. Management and programming organization for sight population of Iran during the next 20 years. Tehran, IR: Ministry of Health and Medical Education; 2005.

11. Moghani SS, Simbar M, Dolatian M, Nasiri M. The relationship between perceived social support and women experiences in menopause. Advances in Nursing \& Midwifery. 2016;25(90).

12. Tarverdy M, Shabani A. Assessment of Psychological Problems In Menopause Women And Relationship's Between Individual And Social Characteristic And Menopausal Symptoms In Referred To Tabriz Educational HospitalS In 2005 2006. Journal of Nursing and Midwifery Urmia University of Medical Sciences. 2007;5(1):37-45.

13. Habibi M, Hanasabzadeh M. The Effectiveness of Mindfulness based Art Therapy on Depression, Anxiety, Stress and Quality of Life among
Postmenopausal Women. Iranian Journal of Ageing. 2014;9(1):22-31.

14. Ford M. A Descriptive Study of Menopausal Woman's Life Experiences, Stressors and Their Occupational Meanings: Eastern Kentucky University Encompass; 2015.

15. Peyrovi H, Jouybary L, Jamshidi Manesh M, Sanagoo A. Women's Experience Of Menopause. Journal of Sabzevar University of Medical Sciences. 1970;16(4):196-205.

16. Cohen LS, Soares CN, Joffe H. Diagnosis and management of mood disorders during the menopausal transition. The American Journal of Medicine. 2005;118(12):93-7. https://doi.org/10.1016/j.amimed.2005.09.042 PMid:16414333

17. Bahri N, Afiat M, Aghamohamadian HR, Delshad Noughabi A, Bahri N. Investigating the Relationship between Severity of Menopausal Symptoms and Depression, Anxiety and Other Menopausal Symptoms. The Iranian Journal of Obstetrics, Gynecology and Infertility. 2013;16(43):14-20.

18. Bener A, Saleh N, Bakir A, Bhugra D. Depression, anxiety, and stress symptoms in menopausal arab women: Shedding more light on a complex relationship. Annals of Medical and Health Sciences Research. 2016;6(4):224.PMid:28480097

19. Henry JD, Crawford JR. The short-form version of the Depression Anxiety Stress Scales (DASS-21): Construct validity and normative data in a large non-clinical sample. British journal of clinical psychology. 2005;44(2):227-39. PMID:16004657

20. Sahebi A, Asghari MJ, Salari R. Validation of Depression Anxiety and Stress Scale (DASS-21) for an Iranian population. Journal of Iranian Psychologists. $2005 ; 1(4)$ : 36-54.

21. Hakimi S, Simbar M, Tehrani FR. Perceived concerns of Azeri menopausal women in Iran. Iranian Red Crescent Medical Journal. 2014;16(5). PMCID:PMC4082510

22. Reed SD, Ludman EJ, Newton KM, Grothaus LC, LaCroix AZ, Nekhlyudov L, et al. Depressive symptoms and menopausal burden in the midlife. Maturitas. 2009;62(3):306-10. https://doi.org/10.1016/j.maturitas.2009.01.002 PMID:19223131

23. Zang H, He L, Chen Y, Ge J, Yao Y. The association of depression status with menopause symptoms among rural midlife women in China. African health sciences. 2016;16(1):97-104. https:// doi.org/10.4314/ahs.v16i1.13 PMID:27358619 PMCID:PMC4915426

24. Rabiee M, Akbari H, Davati A, Moghadamnia 


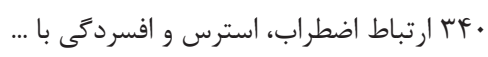

M. Investigating the influence of mood-changes and effective elements of peri menopause on patients' companions referred to hospitals related to Shahed University. The Iranian Journal of Obstetrics, Gynecology and Infertility. 2012;15(15):815 .

25. Abdelrahman RY, Abushaikha LA, al-Motlaq MA. Predictors of psychological well-being and stress among Jordanian menopausal women. Quality of Life Research. 2014;23(1):16773.https://doi.org/10.1007/s11136-013-0464-z PMID:23812907

26. Hakimi S, Simbar M, Ramezani Tehrani F, Zaiery F, Khatami S. Women's perspectives toward menopause: A phenomenological study in Iran. Journal of women \& aging. 2016;28(1):80-9. https://doi.org/10.1080/08952841.2014.954502 PMID:26735699

27. Sanagou A, Jouybari L. A Phenomenological Study of Turkmen Women's in Menopause Experience. The Iranian Journal of Obstetrics, Gyne- cology and Infertility. 2013;15(35):1-10.

28. Hvas L. Menopausal women's positive experience of growing older. Maturitas. 2006;54(3):245-51. https://doi.org/10.1016/j.maturitas.2005.11.006 PMID: 16413977

29. Adler SR, Fosket JR, Kagawa-Singer M, McGraw SA, Wong-Kim E, Gold E, et al. Conceptualizing menopause and midlife: Chinese American and Chinese women in the US. Maturitas. 2000;35(1):11-23. https://doi.org/10.1016/ S0378-5122(00)00090-6

30. Bener A. Depression, anxiety and stress [DASS21] symptoms in menopausal Arab women: Shedding more light on a complex relationship. European Psychiatry. 2016;33:119. https:// doi.org/10.1016/j.eurpsy.2016.01.136

31. Simon JA, Reape KZ. Understanding the menopausal experiences of professional women. Menopause. 2009;16(1):73-6.https://doi.org/10.1097/ gme.0b013e31817b614a PMID: 18779760 